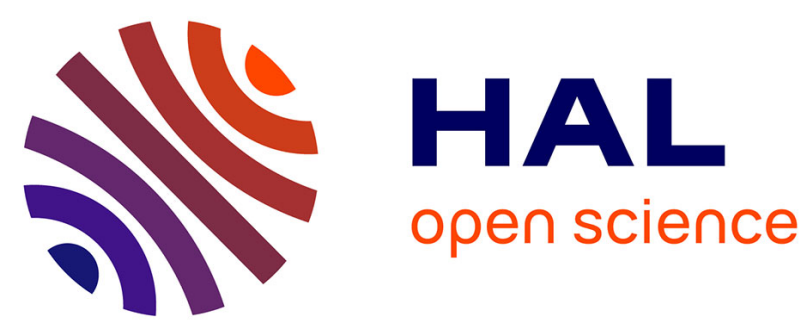

\title{
Low fluence rate photodynamic therapy combined with intravitreal bevacizumab for neovascular age related macular degeneration.
}

Ciro Costagliola, Mario R Romano, Michele Rinaldi, Robeto Dell'Omo, Flavia Chiosi, Massimo Menzione, Francesco Semeraro

\section{To cite this version:}

Ciro Costagliola, Mario R Romano, Michele Rinaldi, Robeto Dell'Omo, Flavia Chiosi, et al.. Low fluence rate photodynamic therapy combined with intravitreal bevacizumab for neovascular age related macular degeneration.. British Journal of Ophthalmology, 2010, 94 (2), pp.180-n/a. 10.1136/bjo.2009.159343 . hal-00508659

\section{HAL Id: hal-00508659 \\ https://hal.science/hal-00508659}

Submitted on 5 Aug 2010

HAL is a multi-disciplinary open access archive for the deposit and dissemination of scientific research documents, whether they are published or not. The documents may come from teaching and research institutions in France or abroad, or from public or private research centers.
L'archive ouverte pluridisciplinaire HAL, est destinée au dépôt et à la diffusion de documents scientifiques de niveau recherche, publiés ou non, émanant des établissements d'enseignement et de recherche français ou étrangers, des laboratoires publics ou privés. 
Title: Low fluence rate photodynamic therapy combined with intravitreal bevacizumab for neovascular age related macular degeneration.

Authors: Ciro Costagliola ${ }^{1}$, Mario R. Romano ${ }^{1,2}$, Michele Rinaldi ${ }^{3}$, Roberto dell'Omo ${ }^{1}$, Flavia Chiosi $^{3}$, Massimo Menzione ${ }^{3}$, Francesco Semeraro ${ }^{4}$.

${ }^{1}$ Dipartimento di Scienze per la Salute, Università degli Studi del Molise, Campobasso, Italy ${ }^{2}$ St Paul's Eye Unit, Royal Liverpool University Hospital, L7 8XP, Liverpool, UK.

${ }^{3}$ Dipartimento di Oftalmologia, Seconda Università degli Studi di Napoli, Napoli, Italy

${ }^{4}$ Dipartimento di Oftalmologia, Università degli Studi di Brescia, Brescia, Italy

*Corresponding author:

Dr. Mario Romano, Dipartimento di Scienze per la Salute, Via F. De Sanctis, 86100 Campobasso Italy, email: romanomario@email.it, phone +393403954704, fax +39 0874 404778

\section{Key words:}

Age-related macular degeneration; bevacizumab, classic choroidal neovascularization, predominantly classic choroidal neovascularization, low fluence PDT

Word count: 2490 
The Corresponding Author has the right to grant on behalf of all authors and does grant on behalf of all authors, an exclusive licence (or non exclusive for government employees) on a worldwide basis to the BMJ Publishing Group Ltd to permit this article (if accepted) to be published in the British Journal of Ophthalmology and any other BMJPGL products and sublicences such use and exploit all subsidiary rights, as set out in our licence.

\section{Competing Interest: None declared.}




\begin{abstract}
Aims: to report efficacy and safety of intravitreal bevacizumab (IVB) alone versus IVB plus low fluence PDT in age-related macular degeneration (AMD) patients and to verify the occurrence of a synergistic effect of the combined approach on visual acuity, size and morphology of lesion, as well as on the treatment rate.

Method: prospective comparative interventional study on 85 patients with treatment naive classic, or predominantly classic, subfoveal choroidal neovascularisation (CNV) secondary to AMD. Patients were randomly assigned to Group 1 (IVB injections) and Group 2 (IVB plus low fluence PDT). In Group 2 the PDT with verteporfin was delivered with low fluence rate $\left(300 \mathrm{~mW} / \mathrm{cm}^{2}\right.$ for 83 seconds, $\left.25 \mathrm{~J} / \mathrm{cm} 2\right)$. The follow-up was scheduled at 1-,3-,6-, 9- and 12-months.

Results: The eye without recurrence received a mean of 2.8 (Group1) vs 1.4 (Group2) IVB injections, whereas the eyes with recurrence received a mean of 3.2 (Group1) vs 2.2 (Group2) IVB injections. The difference in re-injections rate between the two Groups was statistically significant $(P=0.03$, ANOVA test). Visual acuity improvement was not statistically significant between the two Groups $(P=0.31)$.

Conclusion: The combination of IVB with low fluence PDT for the treatment of classic or predominantly classic neovascular AMD works in a synergistic fashion with a significant reduction of IVB re-injections rate.
\end{abstract}




\section{Introduction}

The Macular Photocoagulation Study (MPS) provides guidelines for the evaluation and management of patients with choroidal neovascularization (CNV) secondary to age-related macular degeneration (AMD), ocular histoplasmosis, and idiopathic choroidal neovascularization. ${ }^{1}$ According to the study, eyes with welldemarcated areas of extrafoveal or juxtafoveal classic CNV, as defined by fluorescein angiography, had a better visual prognosis when treated with laser photocoagulation than when managed by observation. ${ }^{1}$ The subgroup analysis of this trial showed the importance of lesion component and size in treatment outcomes.

Photodynamic therapy (PDT) was the recommended treatment by the TAP study Group for patients affected by predominantly classic choroidal neovascularization (CNV) from AMD, with a benefit consisting in a vision loss fewer than 15 letters, over a period of 24 months. ${ }^{2,3,4}$

Although the clinical outcomes of verteporfin therapy have been established, the effects on choroid perfusion have revealed a significant reduction within the entire photosensitized area. ${ }^{5}$ The tissue hypoperfusion is the common denominator and the major trigger of local angiogenesis stimulators, ${ }^{6}$ inhibitors and proteolytic enzymes cooperatively participate in the progressive retinal pigment epithelium defect and growth of choroidal neovascular membranes in AMD. ${ }^{7}$ Therefore the vaso-occlusive mechanism induced by PDT, that affected both CNV and the normal choroids, ${ }^{8}$ may produce an angiogenic response with enhanced expression of vascular endothelial growth factor (VEGF) in human eyes. ${ }^{9}$

Intravitreal injection of a full-length humanized anti-VEGF antibody, as 
Bevacizumab (IVB), has been associated with improvements in visual acuity and reduction of both central retinal thickness (CRT) and angiographic leakage in AMD. ${ }^{10}$ Nevertheless, monotherapy with Bevacizumab requires multiple intravitreal injection, usually with a 4-week-interval, to maintain visual gain. ${ }^{11}$

Recently, PDT with a reduced light dose, to avoid choroid secondary change, combined with intravitreal injection of an anti-angiogenesis compounds, has been suggested as an effective option for AMD in terms of visual improvement, CRT diminution as well as reduced re-treatment rate. ${ }^{12,13}$

Herein we report a comparative consecutive case series of AMD patients treated with IVB alone versus IVB plus low fluence PDT to verify the synergistic effect, if any, of the combined treatment on visual acuity, size and morphology of lesion, as well as on the re-treatment rate.

\section{Patient and Methods}

Study Design

A prospective comparative interventional study on 85 consecutive eyes of 85 patients (38: 47; male: female) aged 50 years or older has been performed. All subjects had a classic or predominantly classic subfoveal CNV secondary to AMD. The presence of blood had to account for $<50 \%$ of the total area occupied by the subfoveal lesion. They were referred to one of the two centres (Università degli Studi di Brescia, Italy; Seconda Università degli Studi di Napoli, Italy) during the period between June 2007 and January 2008. The two local Human Subjects Review Committees (Brescia and Napoli) approved the project. 


\section{Patients and preoperative findings}

The inclusion criteria consisted of, naive classic, or predominantly classic, subfoveal $\mathrm{CNV}$, lesion size of $>2$ disk areas, lesions not exceeding 12 total disk areas (including blood, scar or atrophy, in presence of active neovascularization) and visual acuity in the fellow eye of 20/800 or better.

The exclusion criteria were: age younger than 50 years, severe systemic disease, pregnancy, any uncontrolled ocular disease and presence occult or minimally classic lesions, scarring or atrophy within the lesion.

\section{Materials}

Data collected from each recruited patient included diagnosis, visual acuity, fluorescein (FA) and indocyanine green angiography (ICG) angiographies, intraocular pressure and number of IVB injections. Best corrected visual acuity determination (BCVA), FA, ICG and optical coherence tomography (OCT) were recorded at baseline and, then, repeated the day before the IVB injection and at 1-,3-,6-, 9- and 12-month of follow-up. BCVA was determined with the ETDRS chart at distance of $4 \mathrm{~m}$. FA and ICG (Heidelberg Retina Angiograph 2, Heidelberg, Germany) confirmed eligibility and appropriate classification of the lesions, which were graded according to the MPS system ${ }^{1}$. Only patients with classic and predominantly classic lesions were recruited. Lesions were further investigated with OCT (OCT Stratus, Carl Zeiss Meditec, Dublin, CA). Linear scans of $6 \mathrm{~mm}$ length at $0^{\circ}$ and $90^{\circ}$ were obtained centred on the fovea. Retinal thickness, including intra- and sub-retinal fluid, was measured applying a calibre on the image; therefore the thickness of the neovascular complex was defined as a hyper reflective layer. 
After the patients signed the consent form for the randomization and the identification number was recorded on the baseline data forms, they were randomly assigned to one of the two treatment groups ( 1 and 2 respectively).

At baseline patients included in Group 1 received $1.25 \mathrm{mg}$ IVB. After the first IVB three patients dropped out from the trial and, therefore, they were not included into the study.

The patients of Group 2 were treated at baseline with a $1.25 \mathrm{mg}$ IVB injection followed by PDT within a 2-week period. The PDT with verteporfin was delivered with low fluence rate $\left(300 \mathrm{~mW} / \mathrm{cm}^{2}\right.$ for 83 seconds, light dose of $\left.25 \mathrm{~J} / \mathrm{cm} 2\right)$.

In both Groups, after the first injection, IVB re-injections were scheduled at least four weeks after initial treatment if one of the following criteria was fulfilled: i) drop of BCVA of at least five letters at two repeated tests ii) decrease of BCVA associated with increased leakage of the choroidal neovascularization, as assessed by FA iii) increase of central retinal thickness in OCT of more than $100 \mu \mathrm{m} .{ }^{14}$

The study was carried out for one year and the regimen treatment with IVB re-injections was followed for the first 6 months. After the first 6 months, in case of marked worsening or dissatisfaction, the patients abandoned the study.

\section{Main outcome measure}

The goal of the clinical trial was to verify the efficacy of a combined treatment (IVB + low fluence PDT) compared to IVB alone, in terms of functional results and changes of morphology of choroidal membrane, and advantage in terms of stabilization of the lesions and number of IVB re-treatments. For this purpose, changes in visual acuity, size and thickness of neovascular membrane, and macular thickness at each follow-up check as well the different re-injection treatment rates were recorded. 
Visual outcomes analyzed were change in visual acuity from baseline to each follow up check: a) patients maintaining or gaining vision (gaining $\geq 15$ letters); b) patients with moderate vision loss (loss of less than 15 ETDRS letters); c) patients with severe vision loss (measured as a losing of $\geq 15$ ETDRS letters); d) risk of progression to legal blindness in the study eye (Snellen equivalent visual acuity of 20/200 or worse).

\section{Statistical analysis}

Statistical analysis was performed using the SPSS software 10.0 (SPSS, Chicago,IL, USA). A statistical ANOVA test was performed for analysis of variance on time difference among the covariates 'number of injections', 'age' and 'entity of initial value'. Unequal variance $t$ test was used to compare the mean change in visual acuity from baseline to 12-months for each treatment Group, and between the two groups. A $P$ value of $\leq 0.05$ was considered to be significant.

Coefficient of correlation ( $\rho$ ) was used to verify the relationship between VA and number of IVB injections. A $P$ value of $\leq 0.05$ was considered to be significant. Spearman's rank correlation coefficient $(\rho)$ was used to describe the difference recorded in OCT parameters. A $P$ value of $\leq 0.05$ was considered to be significant. 


\section{Results}

A total of 85 patients were enrolled and randomly assigned to both treatment groups. At the time of initial diagnosis all the patients presented a classic or predominantly classic subfoveal CNV lesion and no patient had been previously treated. The mean age in the first Group was $65.3(\mathrm{SD}=15)$, in the second Group it was $63.2(\mathrm{SD}=12)(\mathrm{P}=0.2)$. Fortyfive eyes (25 phakic, 20 pseudophakic) were treated with IVB injections (Group 1), whereas 40 eyes (22 phakic, 18 pseudophakic) with IVB plus low fluence PDT (Group 2). Patients were randomly assigned to one of the two Groups. (Table 1)

At baseline the mean ETDRS letter distance visual acuity was $50 \pm 12$ and $55 \pm 14$, respectively, in Group 1 and $2(\mathrm{P}=0.06)$.

Table 1: Demographic Characteristics of Study at baseline

\begin{tabular}{lll}
\hline Characteristic & Group 1 (45 eyes) & Group 2 (40 eyes) \\
\hline No Male/female & $20 / 25$ & $18 / 22$ \\
Mean Age \pm SD & $65.3 \pm 15$ & $63.2 \pm 12$ \\
Phakic / Pseudophakic & $25 / 20$ & $22 / 18$ \\
Mean ETDRS letters \pm SD & $50 \pm 12$ & $55 \pm 14$ \\
(Snellen equivalent) & $(0.20 \pm 0.12)$ & $(0.33 \pm 0.18)$ \\
Angiographic CNV greatest linear diameter & $3.15 \pm 1.2$ & $3.85 \pm 1.6$ \\
\pm SD (mm) & & $321 \pm 115$ \\
Central Macular Thickness \pm SD $(\mu \mathrm{m})$ & $330 \pm 98$ & $123 \pm 35$ \\
Hyper reflective area of CNV \pm SD $(\mu \mathrm{m})$ & $110 \pm 58$ & \\
CNV= Choroidal Neovascularization & & \\
IVB = Intravitreal Bevacizumab & & \\
SD= Standard Deviation & &
\end{tabular}




\section{Functional outcomes}

In Group 1, the mean visual acuity score (number of ETDRS letters read) improved from

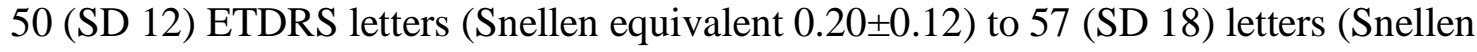
equivalent $0.30 \pm 0.16$ ) at 12 months. At the last check, 21 out of 45 eyes (47\%) gained $\geq$ 15 ETDRS letters, 14 out of 45 eyes (31\%) had lost less than 15 ETDRS letters, 10 out of 45 eyes (22\%) measured as a losing of $\geq 15$ ETDRS letters.

In Group 2, the mean visual acuity improved from 55 (SD 14) ETDRS letters (Snellen equivalent $0.25 \pm 0.19$ ) to 61 (SD 16) letters (Snellen equivalent $0.33 \pm 0.18$ ) at 12 months. At the last check, 14 out of 40 eyes (35\%) gained $\geq 15$ letters, 20 out of 40 eyes (50\%) had lost less than 15 ETDRS letters, 6 out of 40 eyes (15\%) measured as a losing of $\geq 15$ ETDRS letters. (Table 2)

The improvement in visual acuity was greater in Group 2 than that recorded in Group 1, although the difference was not statistically significant $(P=0.31$, unequal variance $t$ test). The difference between baseline and 12-months follow-up VA was statistically significant in each group (Group 1; $P=0.03$; Group 2; $P=0.01$, unequal variance $t$ test). The differences between the two groups were always not significant $(P=0.15$ unequal variance $t$ test ). We did not find any correlation between VA and number of injections performed in both Groups $(\mathrm{R}=0.15, P=0.3)$.

\section{Angiographic outcomes}

The area of CNV and subretinal fibrous tissue/disciform scar remained stable over time on average in both Groups throughout the follow-up period (12-months). In each group the difference of CNV area size between baseline and 12-month check was statistically 
significant (Group 1: $P=0.01$; Group 2: $P=0.001$, ANOVA test), whereas the difference between the two groups was, at the same time, not significant. (Table 2)

(Figure 1, Figure 2)

Optical Coherence Tomography outcomes

The mean change from baseline in centre point thickness was approximately $107 \mu \mathrm{m}$ (SD 35) in Group 1 and $77 \mu \mathrm{m}(\mathrm{SD} 44)$ in Group 2 through 12 months (Group 1: $P=0.002$;

Group 2: $P=0.003$, Spearman test). Overall, the macular thickness significantly decreased through the follow-up period in all subjects.

The hyper reflective area of the neovascular complex remained stable in both Groups during the first 12-months of follow-up. Correlation between baseline and 12-month follow-up macular thickness was statistically significant in both Groups (Group 1: $P=$ 0.002; Group 2: $P=0.001$, Spearman test). (Table 2)

\section{Re-injections Rate}

After the first injection, patients were re-injected if recurrence was noted for an average

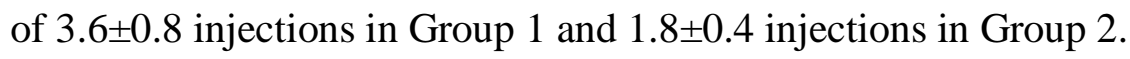

In total, at last check, in Group 1: 2 eyes (4.4\%) had 2 IVB injections, 37 eyes $(82,2 \%)$ had 3 IVB injections, 5 eyes $(11 \%)$ had 4 IVB injections and 1 eye $(2,2 \%)$ had more then 4 injections. In Group 2: 3 eyes (7.5\%) had 2 IVB injections, 33 eyes (82,5\%) had 2 IVB injections, 4 eyes (10\%) had 3 IVB injections. (Table 2)

In Group 1, eyes with recurrence received a mean of 3.2 injections and eyes without recurrence received a mean of 2.8 injections. In Group 2 eyes with recurrence received a mean of 2.2 injections and eyes without recurrence received a mean of 1.4 injections The difference in re-injections rate between the two Groups with $(P=0.035$, ANOVA test $)$ 
and without recurrence ( $P=0.03$, ANOVA test) was statistically significant. (Table 2 )

Neither statistically significant changes in IOP, nor anterior chamber inflammation, retinal detachment, or endophthalmitis were documented. No patient developed myocardial infarction or thromboembolic event.

Table 2 . Results at 12-months follow-up in Group 1 (IVB alone) vs Group 2 (IVB+PDT with low fluence rate)

\begin{tabular}{lccc}
\hline & Group 1 (45 eyes) & Group 2 (40 eyes) & $P$ value \\
\hline Mean ETDRS letters \pm SD (Snellen equivalent) & $57 \pm 18(0.30 \pm 0.16)$ & $61 \pm 16(0.33 \pm 0.18)$ & 0.31 \\
$\quad$ Gained $\geq 15$ ETDRS & $21(47 \%)$ & $14(35 \%)$ & 0.08 \\
$\quad$ Lost $\leq 15$ ETDRS & $14(31 \%)$ & $20(50 \%)$ & 0.09 \\
$\quad$ Lost $\geq 15$ ETDRS & $10(22 \%)$ & $6(15 \%)$ & 0.12 \\
Angiographic CNV greatest linear diameter \pm SD $(\mathrm{mm})$ & $2.80 \pm 0.75$ & $2.65 \pm 1.2$ & 0.35 \\
Central Macular Thickness \pm SD $(\mu \mathrm{m})$ & $223 \pm 72$ & $244 \pm 60$ & 0.21 \\
Hyper reflective Thickness of CNV \pm SD $(\mu \mathrm{m})$ & $78 \pm 24$ & $81 \pm 15$ & 0.15 \\
Mean no. of IVB with recurrence & 3.2 & 2.2 & 0.035 \\
Mean no. of IVB without recurrence & 2.8 & 1.4 & 0.03 \\
CNV Choroidal Neovascularization & & & \\
IVB = Intravitreal Bevacizumab & & & \\
SD $=$ Standard Deviation & & &
\end{tabular}




\section{Discussion}

Combined intravitreal bevacizumab and PDT for AMD effectively maintains or improves VA and reduces the number of re-treatments needed to achieve a vision stabilization, even at 12 months of follow-up. In 2006, Dhalla et al reported a retrospective series of 24 eyes with juxtafoveal or subfoveal CNV secondary to AMD treated with PDT and $1.25 \mathrm{mg}$ of IVB. ${ }^{15}$ All patients were naïve to treatment and had either treatment within a 14-day interval. At 7-month follow-up, 20 out of $24(83 \%)$ patients had stabilization of visual acuity, 16 out of $24(67 \%)$ had improvement in visual acuity. Fifteen eyes (63\%) required only a single combined treatment for CNV resolution. $^{15}$

In a prospective case series of 14 eyes, Ahmadieh et al reported the efficacy of combined single-session with PDT and IVB injection for treatment of neovascular AMD. ${ }^{16}$ A second IVB injection was performed based on fluorescein angiographic evidence of CNV leakage in 13 out 14 eyes (93\%) with a mean interval of $16.3+/-5.9$ weeks. At a mean follow-up of 52.4+/-15.2 weeks the BCVA improved from 0.80+/-0.42 $\log$ MAR to $0.62+/-0.47 \log$ MAR $(\mathrm{p}=0.006)$ and the mean central macular thickness reduced from $308+/ 88 \mu \mathrm{m}$ to $186+/-53 \mu \mathrm{m}(P=0.003)$. The author concluded that the combination therapy with single-session PDT and IVB can improve vision and reduce CMT in neovascular AMD. Repeat IVB injections may maintain the visual gain from the initial combination therapy. ${ }^{16}$

Photodynamic therapy combined with IVB tends to be more effective compared to the monotherapy by reducing the post-PDT increased expression of VEGF-A, inflammatory mediators and reactive oxygen species. ${ }^{18}$ On the other hand, the 
simultaneous combination of PDT with bevacizumab in patients with neovascular AMD may enhance the photochemical stress in normal choroid with prolonged and magnified hypofluorescence in ICG, due to ischemia in normal choriocapillaries.

Recently, Rouvas et al reported the case of enlargement of post PDT spot in a patient with retinal angiomatous proliferation that was treated with PDT combined with an IVB injection. The post PDT hypofluorescent treatment spot had an enlargement from $1,4 \mu \mathrm{m}$ at the day of the treatment, to $5,3 \mu \mathrm{m}$ at the 6-week visit that still was persisting at the 10-month follow-up. ${ }^{17}$

In light of such considerations, the IVB combined with a low fluence PDT may avoid the enlargement of hypofluorescent treatment spot reducing the collateral alteration of the physiologic choroids. Our comparative case series of AMD patients showed a synergistic effect of the combined treatment on the visual acuity, size and morphology of the lesions, as well as reduction of treatment rates.

These findings of ours demonstrate that the improvement in visual acuity was greater in Group 2 than in Group 1, although the difference was not statistically significant $(P=$ 0.31, t-test of unequal variance). The difference between $\mathrm{CNV}$ area size recorded at baseline and at the 12-month check was statistically significant in both Groups (Group 1: $P=0.01$; Group 2: $P=0.001$ ) as well as the macular thickness (Group 1: $P=0.002$; Group 2: $P=0.001$ ).

The main difference between the two groups was the IVB re-injections rate. The eye without recurrence received a mean of 2.8 (Group 1) vs 1.4 (Group 2) IVB injections, whereas the eyes with recurrence received a mean of 3.2 (Group 1) vs 2.2 (Group 2) IVB injections. At the end of follow-up such difference between the two Groups was 
statistically significant $(P=0.03$, ANOVA test). We did not find any correlation between VA and the number of injections performed in both Groups.

PDT is an established modality for the treatment of neovascular AMD, although the release of a wide variety of potent mediators, including vasoactive substances, components of the complement and clotting cascades, acute phase proteins, proteases, peroxidases, radicals, leukocyte chemoattractants, cytokines, growth factors, and other immunoregulators has been documented after its use ${ }^{18-20}$. All these compounds, together with the PDT induced hypoxia, increase VEGF-A levels, as suggested by SchmidtErfurth et al. ${ }^{9}$ Therefore, IVB plus PDT treatment would both ablate established vessels (PDT) and inhibit re-growth due to increased expression of VEGF-A (bevacizumab).

The overall improvement in vision with a good efficacy on fluorescein leakage from $\mathrm{CNV}$ and less IVB re-injections rate throughout the study suggest that a possible synergistic effect may arise from the combination of intravitreal bevacizumab with low fluence PDT for the treatment of classic or predominantly classic neovascular AMD.

These present findings are promising; in fact, the vessel regression effects observed in the present study suggest that the combined use of IVB plus PDT may provide a beneficial option for any ocular disease in which CNV plays a major role. However, the mode, dosage, and timing of administration of these agents in combination may have to be considered to ensure optimal efficacy, as recently pointed out by Ju and coworkers, ${ }^{18}$ who have found that pre-treatment with anti VEGF before PDT resulted in reduced efficacy of PDT, due to the influence of anti VEGF agents both on the inflammatory component of PDT, and on the delivery of verteporfin to the sites of CNV, hence reducing the effective concentration of the photoactive and thereby the efficacy of the 
treatment.

\section{References}

1. Visual outcome after laser photocoagulation for subfoveal choroidal neovascularization secondary to age-related macular degeneration. The influence of initial lesion size and initial visual acuity. Macular Photocoagulation Study Group. Arch Ophthalmol 1994; 112(4): 480-488.

2. Bressler NM. Photodynamic therapy of subfoveal choroidal neovascularization in age-related macular degeneration with verteporfin: two-year results of 2 randomized clinical trials-tap report 2. Arch Ophthalmol 2001; 119(2): 198-207.

3. Ruiz-Oliva F, Cortes J. Verteporfin in Photodynamic Therapy (VIP) Study group. Ophthalmology 2002; 109(6): 1043; author reply 1043-1044.

4. Photodynamic therapy of subfoveal choroidal neovascularization in age-related macular degeneration with verteporfin: one-year results of 2 randomized clinical trials--TAP report. Treatment of age-related macular degeneration with photodynamic therapy (TAP) Study Group. Arch Ophthalmol 1999; 117(10): 1329-1345.

5. Dewi NA, Yuzawa M, Tochigi K, Kawamura A, Mori R. Effects of photodynamic therapy on the choriocapillaris and retinal pigment epithelium in the irradiated area. Jpn J Ophthalmol 2008; 52(4): 277-281.

6. Ohtaki H, Fujimoto T, Sato T et al. Progressive expression of vascular endothelial growth factor (VEGF) and angiogenesis after chronic ischemic hypoperfusion in rat. Acta Neurochir Suppl 2006; 96: 283-287.

7. Steen B, Sejersen S, Berglin L, Seregard S, Kvanta A. Matrix metalloproteinases and metalloproteinase inhibitors in choroidal neovascular membranes. Invest Ophthalmol Vis Sci 1998; 39(11): 2194-2200.

8. Schmidt-Erfurth U, Michels S, Barbazetto I, Laqua H. Photodynamic effects on choroidal neovascularization and physiological choroid. Invest Ophthalmol Vis Sci 2002; 43(3): 830-841.

9. Schmidt-Erfurth U, Schlotzer-Schrehard U, Cursiefen C, Michels S, Beckendorf A, Naumann GO. Influence of photodynamic therapy on expression of vascular endothelial growth factor (VEGF), VEGF receptor 3, and pigment epitheliumderived factor. Invest Ophthalmol Vis Sci 2003; 44(10): 4473-4480. 
10. Krebs I, Lie S, Stolba U, Zeiler F, Felke S, Binder S. Efficacy of intravitreal bevacizumab (Avastin) therapy for early and advanced neovascular age-related macular degeneration. Acta Ophthalmol 2008.

11. Fong KC, Kirkpatrick N, Mohamed Q, Johnston RL. Intravitreal bevacizumab (Avastin) for neovascular age-related macular degeneration using a variable frequency regimen in eyes with no previous treatment. Clin Experiment Ophthalmol 2008; 36(8): 748-755.

12. Sacu S, Varga A, Michels S et al. Reduced fluence versus standard photodynamic therapy in combination with intravitreal triamcinolone: short-term results of a randomised study. Br J Ophthalmol 2008; 92(10): 1347-1351.

13. Smith BT, Dhalla MS, Shah GK, Blinder KJ, Ryan EH, Jr., Mittra RA. Intravitreal injection of bevacizumab combined with verteporfin photodynamic therapy for choroidal neovascularization in age-related macular degeneration. Retina 2008; 28(5): 675-681.

14. Aisenbrey S, Ziemssen F, Volker M et al. Intravitreal bevacizumab (Avastin) for occult choroidal neovascularization in age-related macular degeneration. Graefes Arch Clin Exp Ophthalmol 2007; 245(7): 941-948.

15. Dhalla MS, Shah GK, Blinder KJ, Ryan EH, Jr., Mittra RA, Tewari A. Combined photodynamic therapy with verteporfin and intravitreal bevacizumab for choroidal neovascularization in age-related macular degeneration. Retina 2006; 26(9): 988993.

16. Ahmadieh H, Taei R, Soheilian M, Riazi-Esfahani M, Ahadi H. Single-session photodynamic therapy combined with intravitreal bevacizumab for neovascular age-related macular degeneration. Eur J Ophthalmol 2008; 18(2): 297-300.

17. Rouvas AA, Papakostas TD, Ladas ID, Vergados I. Enlargement of the hypofluorescent post photodynamic therapy treatment spot after a combination of photodynamic therapy with an intravitreal injection of bevacizumab for retinal angiomatous proliferation. Graefes Arch Clin Exp Ophthalmol 2008; 246(2): 315318.

18. Jo N, Mailhos C, Ju M et al. Inhibition of platelet-derived growth factor B signaling enhances the efficacy of anti-vascular endothelial growth factor therapy in multiple models of ocular neovascularization. Am J Pathol 2006; 168(6): 20362053. 


\section{Legend:}

Figure 1: Predominantly classic CNV lesion treated with 4 intravitreal bevacizumab injections during the $12 \mathrm{mo}$ follow-up. The angiogram shows a regression of choroidal neovascular activity from baseline (A) to 12-mo follow-up (B).

Figure 2: Classic CNV lesion treated with low fluence PDT and 2 intravitreal bevacizumab injections. The angiogram shows a regression of choroidal neovascular activity from baseline (A) to 12-mo follow-up (B). 


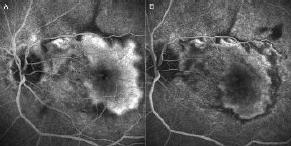


